\title{
BL Eridani: An unstable W Ursae Majoris system with spotted components
}

\author{
Qingyao Liu ${ }^{1,2}$, B. Soonthornthum ${ }^{3}$, Yulan Yang ${ }^{1,2}$, Shenghong Gu ${ }^{1}$, S. Niparugs ${ }^{3}$, M.L. Aniwat Sooksawat ${ }^{3}$, \\ Bi Wang ${ }^{1}$ and M. Naksata ${ }^{3}$ \\ 1 Yunnan Observatory, Academia Sinica, Kunming, China \\ 2 United Laboratory for Optical Astronomy, Chinese Academy of Sciences, China \\ 3 Department of Physics, Faculty of Science, Chiang Mai University, Chiang Mai 50200, Thailand
}

Received August 25, 1995; accepted January 30, 1996

\begin{abstract}
New complete $B V$ light curves of BL Eri are presented. Light-curve variability and asymmetry are seen in both $B$ and $V$ bands, and the observed times of light minimum indicate period changes. The light curves have been analyzed by using the light-curve-synthesis method. Our photometric solution reveals that BL Eri is a contact binary with a common convective envelope. An examination of the available photometric and spectroscopic data indicates that the light-curve variability may be due to changes of the component radii and the light-curve asymmetry may be consistent with starspot activity on one or both components.
\end{abstract}

Key words: stars: binaries: close binaries: individual: BL Eri

\section{Introduction}

Theoretical studies of contact binaries have shown that the evolution of these systems is guided by their stability properties and the presence of dynamic phenomena. Orbital period changes and light-curve variations in contact binaries are observable effects on their stability properties. Therefore, our observational programme includes several binaries, which belong to the dynamically unstable systems in the $M_{1}-M_{2}$ diagram. The first programme object is BL Eri.

The first photoelectric photometric observations of BL Eri were obtained in December 1980 and January 1981 by Kern \& Bookmyer (1986), who proposed a new ephemeris from their photoelectric observations. Yamasaki et al. (1988) presented for the first time and analyzed photoelectric light curves and radial-velocity curves of BL Eri. According to Yamasaki et al. (1988), BL Eri is an almost contact system with both components being noncontact but nearly filling their respective critical Roche lobes. Both components are undermassive for their radii and luminosities, indicating a post-main-sequence evolutionary state of the system.

In this study, we present new $B V$ photoelectric light curves, analyze the observational data, and discuss the system dynamical unstability.

Send offprint requests to: Qingyao Liu

\section{Observations}

Photoelectric photometric observations in $B$ and $V$ bands were made during two nights in December, 1991 with the 106-cm Cssegrain reflector of Yunnan Observatory in China. An EMI 6256B photomultiplier was used in the observations. The following two filters were employed: a Corning 5030 of stock thickness cemented with a $2 \mathrm{~mm}$ Schott GG-13 for the blue region, and a Corning 3389 of stock thickness for the yellow region. A circular diaphragm of $32 \operatorname{arcsec}$ and an integration time of $20 \mathrm{~s}$, for both stars and sky measurements were used. BD- $12^{\circ} 0814$ and BD$12^{\circ} 0821$ were chosen as comparison star and check star, respectively. These stars were alternatively measured in between the observations of the binary. Nightly extinction coefficients were determined from observations of the comparison star. Since the program star and the comparison star are very close to each other (within 16 arcmin), the differential extinction corrections were very small. The magnitude differences between the check and comparison star were constant within probable errors of \pm 0.012 mag. $(V)$ and \pm 0.016 mag. $(B)$ during the observations. A total of 254 and 248 observations in $B$ and $V$ band, respectively, were obtained for BL Eri. Differential $V$ and $B$ magnitudes, in the sense of variable minus comparison star, are presented in Table 1 ( $V$ band) and Table $2(B$ band). 
From our observations, the times of a primary and a secondary minimum were derived by means of a least squares analysis (Table 3). Ephemerides of BL Eri were given by Kern \& Bookmyer (1986) and Yamasaki et al. (1988). We find that these ephemerides given do not fit all the observations. Yamasaki et al. (1988) suspected that the orbital period of BL Eri did likely change between 1980 and 1986. However, in their study no discussion is made about the stability of the orbital period since the ephemeris of BL Eri could not be determined accurately (yamasaki et al. 1988). In this study, we calculate a new ephemeris, by combining our times of minima with those given by Kern \& Bookmyer (1986) and Yamasaki et al. (1988), as

$$
\begin{aligned}
\text { Min.I }=\mathrm{JD}(\text { hel }) 2444606.5884 & +0.41691591 * E \\
\pm 0.0003 & \pm 0.00000012 \text { (p.e.) }
\end{aligned}
$$

The $\mathrm{O}-\mathrm{C}$ values listed in Table 3 , which were caculated with our new ephemeris, reveal that the orbital period of BL Eri has actually changed between 1980 and 1991. Since the observed times of light minimum are too few, the characteristic of the period changes is unclear.

The light variations of BL Eri relative to BD- $12^{\circ} 0814$ are shown in Fig. 1 as the open circles. The photometric disturbance in the present light curves is apparent as those in the observations obtained by Yamasaki et al. (1988). The maximum brightness at phase $0.75 \mathrm{p}$ was found to be fainter by $0.038 \operatorname{mag}(V)$ and $0.042 \operatorname{mag}(B)$ than that at phase $0.25 \mathrm{p}$. The notable disturbance occured in phase from $0.62 \mathrm{p}$ to $0.92 \mathrm{p}$, so the primary minimum showed such an asymmetry that the descending branch is fainter than the ascending branch. In order to compare with the 1982/1986 observations published by Yamasaki et al. (1988), we showed their observations in Fig. 1, too, as the filled circles. Since different comparison stars were used in 1982/1986 and 1991 observations, a vertical shift was applied to Yamasaki et al.'s (1988) observations to match our observations. It is evident that the light curves of $\mathrm{BL}$ Eri have changed in the time interval between 1982/1986 and 1991. The amplitude of the light curves in 1991 has increased by $0.06 \mathrm{mag} .(V)$ and $0.04 \mathrm{mag}$. $(B)$ as compared with the 1982/1986 observations. Within the observation errors, the variations of the amplitudes of both Min.I minus Max.I and Min.II minus Max.II are almost the same, so there are two different suggestions to explain these variations of the amplitudes: luminosities of both components increased or inclination of the orbit increased from 1982 to 1991. More discussion will be made in the last section.

\section{Photometric solution}

Photometric solutions of BL Eri were obtained by using the 1992 version of the Wilson-Devinney program (Wilson 1992). The individual observations were combined into 54 normal points in each color; the number of individual points in each normal points was taken as the weight

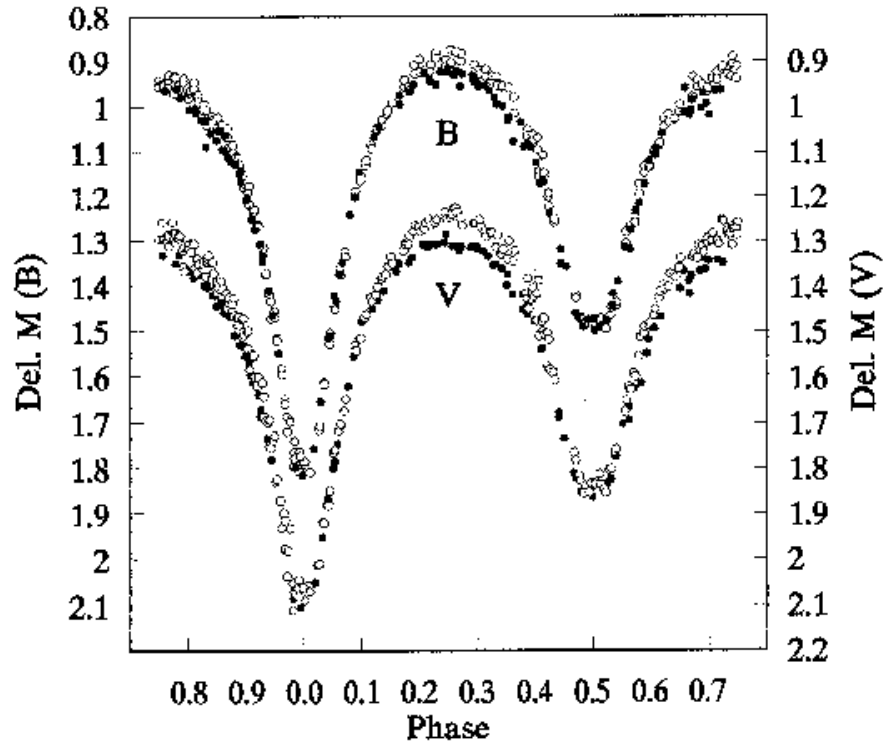

Fig. 1. The light curves of BL Eri from the present observations (open circles) and Yamasaki's et al. observations in 1982 and 1986 (filled circles)

of each point. The two light curves were used simultaneously in deriving our solutions. We adopted a temperature for star 1 (star eclipsed at Min.I) of $5980 \mathrm{~K}$, which corresponds to a spectral type G0V from Yamasaki et al.'s analyses of the spectrum and color indices. Gravity-darkening exponents, $g_{1}, g_{2}=0.32$, and the bolometric albedo of the two components, $A_{1}, A_{2}=0.50$ were assumed. The reflection effect was computed with the detailed model of Wilson (1990), and linear limb darkening laws were used. The mass ratio $(q=0.54)$ of BL Eri was determined from a few spectral measurements (Yamasaki et al. 1988), but an extensive search for a reliable mass ratio $q$ in the photometric solutions is still necessary. Thus solutions for several assumed values of mass ratio $q(0.2,0.3,0.4,0.5,0.6$, $0.7,0.8,0.9)$ were obtained. Because the transit eclipse occurs at the primary minimum according to Yamasaki et al.'s spectroscopic observations (Yamasaki et al. 1988), no values of $q>1$ were tried. The adjustable parameters employed were: the orbital inclination i), the mean temperature of star $2\left(T_{2}\right)$, the monochromatic luminosity of star $1\left(L_{1}\right)$ derived from the Planck function for the appropriate temperature, and the dimensionless potentials of star 1 and star 2, ( $\Omega 1$ and $\Omega 2$ ).

Assuming initially a detached system, the differential corrections always started from the mode 2 for each assumed value of $q$. A convergent solution for each value of $q$, however, was always achieved in the mode 3 (contact mode). The resulting sums of weighted square deviations $\Sigma$ for each value of $q$ are plotted in Fig. 2. A minimum of $\Sigma$ is achieved at $q=0.5$. At this point, the adjustable parameters were expanded to includ $q$ and $x$ (values of $x$ may slightly differ from theory due to the 
Table 1. $V$ observations for BL Eri

\begin{tabular}{|c|c|c|c|c|c|c|c|c|c|}
\hline \multicolumn{2}{|c|}{$\begin{array}{l}\text { JD (He 1) Del. M } \\
2448600+\end{array}$} & \multicolumn{2}{|c|}{$\begin{array}{l}\text { JD(Hej) Del. } \\
2448600+\end{array}$} & \multicolumn{2}{|c|}{$\begin{array}{l}\text { JD (He l) Del. M } \\
2448600+\end{array}$} & \multicolumn{2}{|c|}{$\begin{array}{l}\text { JD (He l) Del. N } \\
2448600 \text { t }\end{array}$} & \multicolumn{2}{|c|}{$\begin{array}{l}\text { JD (Hel) De l, M } \\
2448600+\end{array}$} \\
\hline 2. 0556 & 1.426 & .1648 & 1. 359 & .2681 & 1. 471 & 0991 & 1.485 & 2087 & 1. 334 \\
\hline .0566 & 1. 449 & .1659 & 1. 337 & .2706 & 1.502 & .1016 & 1.512 & .2098 & 1. 349 \\
\hline .0593 & 1. 411 & .1745 & 1. 334 & .2715 & 1. 498 & .1027 & 1. 509 & .2173 & 1. 321 \\
\hline .0603 & 1.421 & .1754 & 1. 328 & .2743 & 1.550 & .1052 & 1.540 & .2184 & 1. 305 \\
\hline .0626 & 1.475 & .1780 & 1. 338 & .2753 & 1.553 & .1063 & 1. 584 & .2210 & 1. 306 \\
\hline .0643 & 1. 510 & .1790 & 1. 309 & 2778 & 1. 619 & .1090 & 1.556 & .2220 & 1. 286 \\
\hline .0669 & 1.499 & .1815 & 1. 311 & .2791 & 1. 601 & .1100 & 1. 6,00 & .2247 & 1. 277 \\
\hline .0680 & 1.507 & .1825 & 1. 324 & .2816 & 1. 612 & .1172 & 1. 688 & .2260 & 1. 264 \\
\hline .0706 & 1.577 & .1851 & 1. 303 & .2828 & 1. 643 & .1182 & 1.695 & .2286 & 1. 268 \\
\hline .0716 & 1. 583 & 1861 & 1. 290 & .2859 & 1. 700 & .1208 & 1. 752 & .2297 & 1. 277 \\
\hline .0743 & 1.589 & .1883 & 1.299 & .2870 & 1. 694 & .1222 & 1.756 & .2325 & 1.271 \\
\hline .0753 & 1.606 & . 1894 & 1.282 & .2896 & 1.739 & .1251 & 1.829 & .2336 & 1. 258 \\
\hline .0897 & 1.765 & .1922 & 1.297 & .2907 & 1. 730 & .1262 & 1.823 & .2362 & 1.250 \\
\hline . 0907 & 1. 783 & .1933 & 1.305 & .2971 & 1.898 & .1286 & 1.871 & .2374 & 1. 264 \\
\hline .0930 & 1.816 & .1959 & \$. 251 & .2981 & 1. 915 & .1296 & 1.932 & .2403 & 1. 257 \\
\hline .0940 & 1.827 & .1970 & 1. 255 & 3007 & 1. 982 & .1322 & 1.978 & .2414 & 1.250 \\
\hline .0964 & 1.855 & .1995 & 1.278 & .3017 & 1.933 & .1336 & 2.037 & .2474 & 1.235 \\
\hline .0975 & 1.838 & .2005 & 1. 284 & .3042 & 2.112 & 1378 & 2. 052 & .2485 & 1.247 \\
\hline$: 1007$ & 1.853 & .2031 & 1.267 & .3053 & 2.075 & .1389 & 2.070 & .2511 & 1. 244 \\
\hline .1018 & 1. 834 & .2042 & 1. 272 & .3084 & 2. 045 & .1414 & 2.063 & .2523 & 1. 238 \\
\hline .1045 & 1.835 & .2066 & 1.285 & 3.0357 & 1. 283 & .1425 & 2. 081 & .2547 & 1. 224 \\
\hline .1079 & 1.813 & .2076 & 1. 296 & .0369 & 1. 308 & .1450 & 2.061 & .2558 & 1. 235 \\
\hline .1088 & 1.837 & .2138 & 1.286 & .9397 & 1.273 & .1461 & 2.089 & .2584 & 1.246 \\
\hline .1112 & 1.847 & .2148 & 1,288 & .0410 & 1. 257 & .1488 & 2.057 & .2595 & 1. 264 \\
\hline .1122 & 1.854 & .2183 & 1.293 &.$(1435$ & 1. 258 & .15000 & 2.070 & .2678 & 1. 259 \\
\hline .1147 & l. 804 & .2192 & 1.281 & .0446 & 1. 295 & .1558 & 2.012 & 2689 & 1.258 \\
\hline .1165 & 1.801 & .2214 & 1. 286 & .0471 & 1. 299 & .1569 & 2.010 & .2721 & 1. 250 \\
\hline .1189 & 1. 763 & .2225 & 1. 287 & .0483 & 1. 311 & .1593 & 1.920 & .2732 & 1.267 \\
\hline .1199 & 1. 758 & .2247 & 1. 320 & .0508 & 1. 257 & .1603 & 1. 921 & .2758 & 1. 278 \\
\hline .1260 & 1. 674 & .2256 & 1.315 & .0522 & 1. 280 & .1632 & 1.882 & .2770 & 1. 288 \\
\hline .1270 & 1.650 & .2285 & 1. 317 & .0547 & 1.293 & .1643 & 1.84 & .2801 & 1. 279 \\
\hline . 1291 & 1.621 & .2294 & 1.305 & .0557 & 1. 293 & .1668 & 1. 766 & .2812 & 1. 280 \\
\hline .1301 & 1. 627 & .2319 & 1.302 & . 15866 & 1.357 & .1679 & 1.760 & .2838 & 1.313 \\
\hline .1323 & 1.592 & .2329 & 1.306 & .0598 & 1.366 & .1707 & 1. 718 & .2850 & 1.285 \\
\hline .1333 & 1. 598 & .2352 & 1.339 & .0632 & 1.316 & .1718 & 1.703 & .2877 & 1. 329 \\
\hline .1357 & 1.554 & .2362 & 1. 350 & .0643 & 1. 324 & .1745 & 1. 674 & .2889 & 1. 317 \\
\hline .1368 & 1.513 & .2389 & 1.348 & .0671 & 1.359 & .1756 & 1.649 & .2921 & 1.339 \\
\hline .1394 & 1.504 & .2399 & 1. 343 & .0683 & 1.370 & .1821 & 1.532 & .2933 & 1. 298 \\
\hline .1404 & 1.488 & .2422 & 1.337 & .0749 & 1.377 & .1831 & 1.54 & .2963 & 1. 343 \\
\hline .1430 & 1. 486 & .2432 & 1.332 & .0760 & 1. 345 & .1857 & 1.5 & .2975 & 1. 321 \\
\hline . 1440 & 1.480 & .2455 & 1. 382 & .0788 & 1. 362 & .1868 & & .3056 & 1. 415 \\
\hline .1466 & 1.465 & .2466 & 1.370 & .0798 & l. 347 & .1895 & 1.469 & .3067 & 1. 382 \\
\hline .14 & 1.449 & .2493 & 1.391 & .0825 & 1. 406 & .1908 & 1. 46 & .3095 & 1. 427 \\
\hline 15 & 1.425 & .2503 & 1.402 & .0836 & 1. 413 & .1935 & 1.423 & .3106 & 1.403 \\
\hline & $1.41 \mathrm{n}$ & .2561 & 1.415 & .0860 & 1. 388 & .1946 & 1.433 & .3133 & 1.415 \\
\hline & 1.397 & .2577 & 1.423 & .0872 & 1. 405 & .1973 & 1.419 & .3144 & 1.386 \\
\hline & 1.40 & .2600 & 1.439 & .0903 & 1. 447 & .1987 & & .3170 & 1.476 \\
\hline & & .2609 & 1.425 & .0914 & 1. 435 & .2013 & & .3181 & 1.470 \\
\hline & & .2637 & 1.471 & .0941 & 1.454 & .2024 & 1.377 & .3207 & 1. 504 \\
\hline & 1. 375 & .2647 & 1.469 & .0953 & 1. 465 & .2049 & 1. 373 & .3218 & 1. 518 \\
\hline .1622 & 1. 379 & .2672 & 1.472 & .0978 & 1.484 & .2063 & 1.356 & & \\
\hline
\end{tabular}

possible effects of stellar activity). The mass ratio converged to $q=0.536$ in the final solution, which is in very good agreement with the spectroscopic value determined by Yamasaki et al. 1988). Our solution reveals that the system is a contact binary. The photometric parameters are listed in Table 4, and the computed light curves (solid lines) are shown in Fig. 3, together with the corresponding observations (circles).

The theoretical light curves do fit rather satisfactorily with the observations, except at secondary maximum where the disagreement is severe. Unequal quadrature light level, which is called the O'Connell effect, has been observed in many eclipsing binaries and several 
Table 2. $B$ observations for BL Eri

\begin{tabular}{|c|c|c|c|c|c|c|c|c|c|}
\hline $\begin{array}{l}\text { JD (He l) } \\
2448600\end{array}$ & $\begin{array}{l}\text { De } 1 . M \\
+\end{array}$ & $\begin{array}{l}\text { JD (He l) } \\
2448600\end{array}$ & & $\begin{array}{l}\text { J1) (He 1) } \\
2448600\end{array}$ & & $\begin{array}{l}\mathrm{JD} \text { (He 1) } \\
2448600\end{array}$ & $\begin{array}{l}\text { De1. } \\
+\end{array}$ & $\begin{array}{l}\text { JD (Hel) } \\
2448600+\end{array}$ & De 1. M \\
\hline 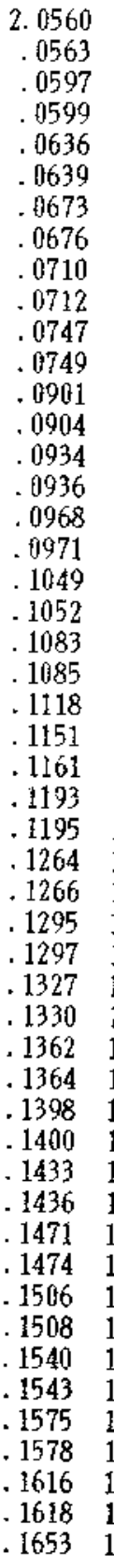 & $\begin{array}{l}1.063 \\
1.080 \\
1.074 \\
1.072 \\
1.143 \\
1.156 \\
1.154 \\
1.147 \\
1.221 \\
1.220 \\
1.258 \\
1.249 \\
1.419 \\
1.425 \\
1.470 \\
1.468 \\
1.486 \\
1.484 \\
1.491 \\
1.487 \\
1.477 \\
1.471 \\
1.493 \\
1.464 \\
1.446 \\
1.440 \\
1.434 \\
1.303 \\
1.278 \\
1.270 \\
1.265 \\
1.245 \\
1.257 \\
1.194 \\
1.172 \\
1.147 \\
1.132 \\
1.135 \\
1.130 \\
1.103 \\
1.097 \\
1.086 \\
1.081 \\
1.045 \\
1.025 \\
1.018 \\
1.034 \\
1.028 \\
1.026 \\
1.012\end{array}$ & $\begin{array}{l}.1655 \\
.1749 \\
.1751 \\
.1784 \\
.1786 \\
.1818 \\
.1821 \\
.1855 \\
.1858 \\
.1887 \\
.1889 \\
.1926 \\
.1929 \\
.1964 \\
.1966 \\
.1999 \\
.2001 \\
.2035 \\
.2038 \\
.2070 \\
.2072 \\
.2142 \\
.2144 \\
.2187 \\
.2189 \\
.2219 \\
.2221 \\
.2251 \\
.2253 \\
.2289 \\
.2291 \\
.2323 \\
.2325 \\
.2356 \\
.2358 \\
.2393 \\
.2395 \\
.2426 \\
.2429 \\
.2459 \\
.2462 \\
.2496 \\
.2499 \\
.2565 \\
.2567 \\
.2604 \\
.2606 \\
.2630 \\
.2644 \\
.2675 \\
\end{array}$ & $\begin{array}{l}.996 \\
.980 \\
.936 \\
.957 \\
.943 \\
.963 \\
.954 \\
.968 \\
.961 \\
.935 \\
.940 \\
.942 \\
.925 \\
.920 \\
.912 \\
.925 \\
.930 \\
.907 \\
.890 \\
.056 \\
.942 \\
.942 \\
.930 \\
.932 \\
.936 \\
.952 \\
.961 \\
.946 \\
.937 \\
.981 \\
.969 \\
.959 \\
.946 \\
.973 \\
.989 \\
1.005 \\
.994 \\
1.030 \\
1.024 \\
1.028 \\
1.035 \\
1.046 \\
1.022 \\
1.065 \\
1.047 \\
1.099 \\
1.084 \\
1.112 \\
1.114 \\
1.164\end{array}$ & 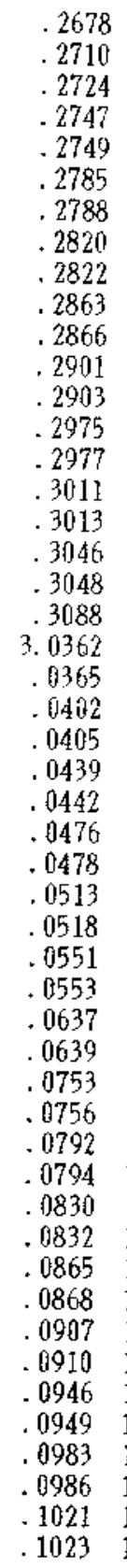 & $\begin{array}{l}1.159 \\
1.192 \\
1.176 \\
1.237 \\
1.226 \\
1.262 \\
1.265 \\
1.315 \\
1.325 \\
1.418 \\
1.426 \\
1.454 \\
1.469 \\
1.649 \\
1.659 \\
1.693 \\
1.698 \\
1.740 \\
1.779 \\
1.773 \\
.909 \\
.935 \\
.939 \\
.911 \\
.948 \\
.953 \\
.959 \\
.942 \\
.933 \\
.969 \\
.972 \\
.950 \\
.968 \\
.964 \\
1.006 \\
1.040 \\
1.020 \\
1.029 \\
1.053 \\
1.062 \\
1.049 \\
1.039 \\
1.093 \\
1.089 \\
1.099 \\
1.120 \\
1.114 \\
1.127 \\
1.179 \\
1.169\end{array}$ & $\begin{array}{l}.1057 \\
.1059 \\
.1094 \\
.1096 \\
.1176 \\
.1178 \\
.1213 \\
.1216 \\
.1255 \\
.1257 \\
.1290 \\
.1292 \\
.1326 \\
.1332 \\
.1382 \\
.1385 \\
.1418 \\
.1421 \\
.1454 \\
.1457 \\
.1493 \\
.1495 \\
.1562 \\
.1565 \\
.1597 \\
.1599 \\
.1636 \\
.1639 \\
.1673 \\
.1675 \\
.1711 \\
.1714 \\
.1749 \\
.1752 \\
.1825 \\
.1827 \\
.1861 \\
.1864 \\
.1900 \\
.1903 \\
.1940 \\
.1942 \\
.1977 \\
.1980 \\
.2017 \\
.2020 \\
.2054 \\
.2059 \\
.2091 \\
.2094 \\
1\end{array}$ & $\begin{array}{l}1.215 \\
1.217 \\
1.234 \\
1.228 \\
1.373 \\
1.370 \\
1.432 \\
1.472 \\
1.517 \\
1.521 \\
1.582 \\
1.595 \\
1.687 \\
1.720 \\
1.796 \\
1.765 \\
1.765 \\
1.809 \\
1.791 \\
1.785 \\
1.808 \\
1.808 \\
1.716 \\
1.708 \\
1.612 \\
1.616 \\
1.528 \\
1.503 \\
1.444 \\
1.451 \\
1.359 \\
1.375 \\
1.324 \\
1.335 \\
1.195 \\
1.187 \\
1.165 \\
1.154 \\
1.130 \\
1.118 \\
1.083 \\
1.091 \\
1.054 \\
1.048 \\
1.041 \\
1.028 \\
1.015 \\
1.009 \\
.991 \\
1.991\end{array}$ & $\begin{array}{l}.2178 \\
.2180 \\
.2214 \\
.2217 \\
.2251 \\
.2254 \\
.2291 \\
.2293 \\
.2330 \\
.2332 \\
.2367 \\
.2369 \\
.2407 \\
.2410 \\
.2478 \\
.2481 \\
.2515 \\
.2518 \\
.2552 \\
.2554 \\
.2588 \\
.2591 \\
.2682 \\
.2685 \\
.2726 \\
.2728 \\
.2763 \\
.2766 \\
.2806 \\
.2808 \\
.2843 \\
.2846 \\
.2882 \\
.2885 \\
.2926 \\
.2928 \\
.2968 \\
.2971 \\
.3061 \\
.3063 \\
.3100 \\
.3102 \\
.3137 \\
.3140 \\
.3175 \\
.3178 \\
.3212 \\
.3214 \\
\end{array}$ & $\begin{array}{r}.957 \\
.941 \\
.957 \\
.966 \\
.908 \\
.928 \\
.896 \\
.924 \\
.926 \\
.919 \\
.923 \\
.907 \\
.881 \\
.893 \\
.911 \\
.896 \\
.904 \\
.875 \\
.880 \\
.879 \\
.900 \\
.883 \\
.944 \\
.908 \\
.920 \\
.928 \\
.917 \\
.935 \\
.942 \\
.934 \\
.934 \\
.953 \\
.957 \\
.955 \\
.956 \\
.960 \\
.979 \\
1.014 \\
1.076 \\
1.050 \\
1.080 \\
1.061 \\
1.094 \\
1.068 \\
1.108 \\
1.106 \\
1.200 \\
1.192\end{array}$ \\
\hline
\end{tabular}

suggestions have been made to explain this effect by various authors, such as the effects of mass exchange and stellar activity. The BL Eri system was non-contact in 1982 (Yamasaki et al. 1988) so that mass exchange does not appear to be a likely mechanism to explain photometric distortions observed in 1982 and 1991. For cool stars, such as the components in BL Eri, dark areas analogous to sunspots appear to be the most probable explanation.
Since the 1992 version of the Wilson-Devinney code offers the option of including spots in the photometric model, the analysis has been pursued to obtain an estimate of the likely properties of the spot(s) producing the photometric perturbations on the light curve of BL Eri.

The observed distortion, which Maximum II being fainter than Maximum I, could be caused by a cool region on either component, so several groups of cool spots 
Table 3. Times of minimum light for BL Eri

\begin{tabular}{|c|c|c|c|c|}
\hline $\begin{array}{l}\text { Heel JD. } \\
2440000+\end{array}$ & Min. & Epoch & $\mathrm{O}-\mathrm{C}$ & source \\
\hline 4603.67091 & 1 & -7 & +0.0009 & Kern and Bookmyer( 1986) \\
\hline 4604.71459 & II & -4.5 & +0.0023 & Kern and Bookmyer( 1986) \\
\hline 4606.58936 & 1 & 0 & +0.0010 & Kern and Boolomyer( 1986) \\
\hline 460763275 & II & 2.5 & +0.0021 & Kern and Bookmyer( 1986) \\
\hline 5298.8745 & It & 1660.5 & -0.0028 & Yamasakt et al.(1988) \\
\hline 5299.917: & 1 & 1663 & -0.0026 & Yamasaki et al.(1988) \\
\hline 5300.9599 & $\|$ & 1665.5 & -0.0019 & Yamasak et al. (1988) \\
\hline 8602.1026 & H & 9583.5 & +0.0006 & present paper \\
\hline 8603.1462 & 1 & 9586 & +0.0009 & present paper \\
\hline
\end{tabular}

Table 4. Photometric solutions for BL Eri

\begin{tabular}{|c|c|c|c|c|}
\hline \multirow[b]{2}{*}{ colont } & \multicolumn{2}{|c|}{ no epot: } & \multicolumn{2}{|c|}{ + epots } \\
\hline & $\mathbf{v}$ & $\mathbf{B}$ & $\mathbf{V}$ & $\mathbf{B}$ \\
\hline$L 1 /(1,1+L 2)$ & $0.7073 \pm 0.0026$ & $0.7223 \pm 0.0031$ & $0.6986 \pm 0.0014$ & $0.7117 \pm 0.0013$ \\
\hline$\times 1(=x 2)$ & 0.570 & 0.750 & 0.550 & 0.740 \\
\hline $\mathbf{i}$ & \multicolumn{2}{|c|}{$86^{\circ} .2 \pm 0.7$} & \multicolumn{2}{|c|}{$89^{\circ} .8 \pm 0.8$} \\
\hline$q=m 2 / m 1$ & \multicolumn{2}{|c|}{$0.536 \pm 0.004$} & \multicolumn{2}{|c|}{$0.546 \pm 0.002$} \\
\hline $\operatorname{A1}(-A)$ & \multicolumn{2}{|c|}{0.500} & \multicolumn{2}{|c|}{0.500} \\
\hline $\mathbf{B}(=\infty)$ & \multicolumn{2}{|c|}{0.320} & \multicolumn{2}{|c|}{0.320} \\
\hline$\Omega \mathbf{1}(=\Omega 2)$ & \multicolumn{2}{|c|}{$2.9202 \pm 0.0058$} & \multicolumn{2}{|c|}{$2.9108 \pm 0.0030$} \\
\hline \% overconthet & \multicolumn{2}{|c|}{15.5} & \multicolumn{2}{|c|}{16.4} \\
\hline$\Sigma(0-c)^{2}$ & \multicolumn{2}{|c|}{0.0095} & \multicolumn{2}{|c|}{0.0020} \\
\hline component & 1 & 2 & 1 & 2 \\
\hline $\mathbf{T}_{\mathbf{M}}$ & 5980 & $5547 \pm 16$ & 5980 & $5603 \pm 7$ \\
\hline $\mathbf{z}(p \circ)$ & $0.4146 \pm 0.0009$ & $0.3158 \pm 0.0019$ & $0.4156 \pm 0.0004$ & $0.3167 \pm 0.0011$ \\
\hline$x(t) d a)$ & $0.4413 \pm 0.0011$ & $0.3312 \pm 0.0023$ & $0.4425 \pm 0.0005$ & $0.3311 \pm 0.0013$ \\
\hline rockit) & $0.4733 \pm 0.0016$ & $0.3692 \pm 0.0040$ & $0.4746 \pm 0.0007$ & $0.3694 \pm 0.0023$ \\
\hline
\end{tabular}

placed on the surface of either component were tried. The derived fits to the observed light curves were better than those found for the unspotted solution, but still not completely satisfactory. In the final solution the assumption was made that cool spots are on both components, because both stars are likely to develop dark-spot activity of the type seen on the Sun. The DC program was used to obtain final spot parameters. Since the cool spots were placed on both the stars, the parameters $q, i, T_{2}$, and $L_{1}$ were varied. The differential corrections were calculated until the adjustment became smaller than their probable errors. The final results of the spotted solution are in- cluded in Table 4 and the final spot parameters are given in Table 5. The theoretical light curves for the spotted solution are shown as solid lines in Fig. 4.

\section{Conclusion and discussion}

From 1982 to 1991, some properties of the system have changed. 1) The orbital period of the system has increased. The times of light minimum derived from the observations are too few to ascertain whether the period change of BL Eri was continuous or had occurred suddenly. The O-C values reveal that the orbital period of BL Eri has increased of less than $0.24 \mathrm{~s}$ from 1982 to 1991. 2) The 


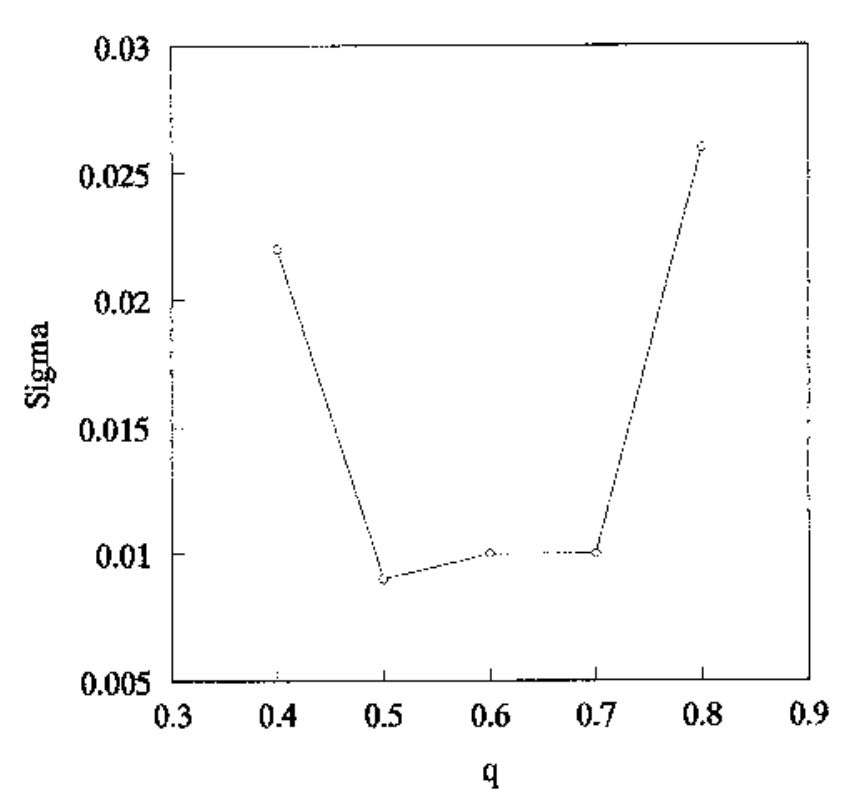

Fig. 2. Variance of the computed fit as a function of the mass ratio $q$

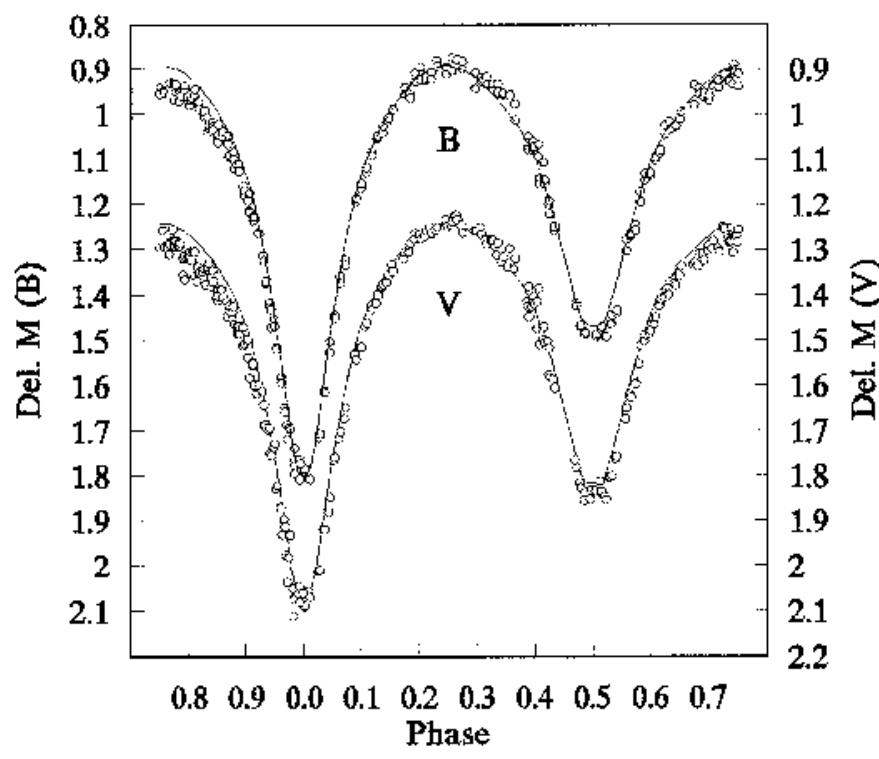

Fig. 3. Observed $B V$ light curves (points) and standard photometric model (solid lines) for BL Eri

Table 5. Spot parameters for BL Eri

\begin{tabular}{ccccc} 
stax & co-latitude & langitnde & ang.radtum & T.F.* \\
1 & $106^{\circ} .390$ & $27^{\circ} .430$ & 9.400 & 0.784 \\
2 & $103^{\circ} .380$ & $255^{\circ} .000$ & 16.460 & 0.700 \\
\hline
\end{tabular}

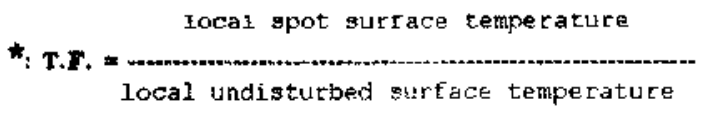

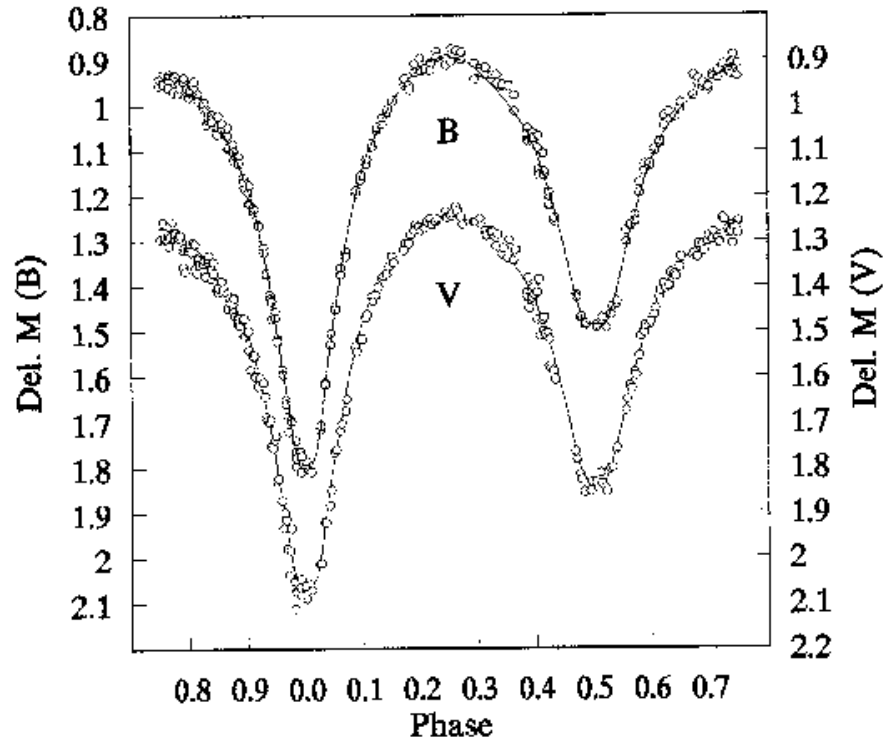

Fig. 4. As Fig. 3, but with dark spots included in the photometric model

luminosities of the system components have raised from 1982 to 1991. As shown in Fig. 1, the amplitudes of the light curves in 1991 has increased by $0.06 \operatorname{mag}(V)$ and $0.04 \mathrm{mag}(B)$, as compared to the 1982 amplitude. Since the orbital inclination of the system and the luminosities at primary and secondary eclipses have not notably changed from 1982 to 1991, the increase of the light curve amplitudes suggests that the luminosities of both components have raised. 3) The radii of both components of BL Eri have also increased so that the almost contact system in 1982 became an over-contact binary in 1991. The masses of both components, which were determined by Yamasaki et al. (1988) from their observational data, are so small that this system is located in the dynamically unstable region of the $M_{1}-M_{2}$ diagram, as determined by Hazlehurst \& Refsdal (1984) and Kahler et al. (1987). The observed variations of the orbital period, luminosities and radii of BL Eri also suggest that it is an unstable contact system. The unstable behaviour of binaries should affect their evolution. As predicted by the "Thermal Relaxation Oscillation Theory" (see Lucy \& Wilson 1979 and references therein), in some evolutionary phase contact binaries would undergo oscillations about a state of marginal contact, and unstable behaviour of such systems should occur in this evolutionary state. For BL Eri, the photometric solutions of the light curves in 1982 and 1991 directly reveal the oscillation behaviour of its geometrical configuration, but also unstable dynamical and thermodynamical properties. Perhaps, BL Eri could be an example of from broken-contact to re-contact systems.

Acknowledgements. We would like to acknowledge the support of the Academia Sinica, the National Science and Committee 
of Yunnan province of China, and the DTC of Thailand under "the China-Thailand cooperation program". QL, SG, and YY would like to thank the Chiang Mai University hospitality during their visit to Thailand, and BS, SN, MLAS, and MN would like to thank the Yunnan Observatory for hospitality during their visit to China.

\section{References}

Claret A., Gimenez A., 1990, A\&A 230, 412
Hazlehurst J., Refsdal S., 1984, A\&A 133, 63

Kahler H., Matraka B., Weigert A., 1987, A\&A 172, 179

Kern J.R., Bookmyer B.B., 1986, IBVS No. 2850

Lucy L.B., Wilson R.E., 1979, ApJ 231, 502

Wilson R.E., 1990, ApJ 356, 613

Wilson R.E., 1992, Revised version of Wilson-Devinney code (Priv. Comm.)

Yamasaki A., Jugaku J., Seki M., 1988, AJ 95, 894 